$(51.23 \%)$ and minor (5.94\%). The numbers of recommendations regarding the drug-drug interactions spotted were: 31 therapeutic exchange (7.67\%), 88 dosage recommendation (21.78\%), 4 adding drug to the treatment $(0.99 \%), 99$ proposal to withdraw a particular drug (24.50\%), 182 monitoring (45.04\%). Total number of food-drug interactions was 286 (1.43 per patient), with 118 instances of intravenous incompatibility warnings made to the ward nurses ( 0.59 per patient). 62 inappropriate drug dosages ( 0.31 per patient) and 3 drugs containing the same active substances in different formulations (0.015 per patient) were reported to the prescribing physicians. In 124 cases (0.62 per patient), pharmacists requested information about the use of the drugs prescribed for treatment.

Upon discussion of the results, the physicians provided feedback and acted according to three options: (9.14\%) the physician didn't agree (they believed the situation didn't require an intervention), $(58.53 \%)$ the physician felt it was sufficient to monitor the patient's status, considering the suggested change in treatment, (32.31\%) the physician agreed to a change in the patient's treatment, applying the recommendation pharmacists made.

Conclusions Our pharmacy department discovered that continuous service at this level of quality is needed for ward patients. Similar studies should be encouraged by health care leaders in Turkey to improve hospital care.

No conflict of interest.

\section{BEA-005 UNLICENSED AND OFF-LABEL DRUG PRESCRIPTION AT DISCHARGE FROM A SWISS CHILDREN'S HOSPITAL}

doi:10.1136/ejhpharm-2013-000276.616

${ }^{1} \mathrm{C}$ Zaugg, ${ }^{1} \mathrm{~J}$ Behringer, ${ }^{2} \mathrm{M}$ Walther, ${ }^{2 \mathrm{M} \text { Köhler. }}$ 'Spitalapotheke, Kantonsspital Aarau; ${ }^{2}$ Klinik für Kinder und Jugendliche, Kantonsspital Aarau

\section{Introduction.}

For children, many drugs are used without marketing authorization ("unlicensed", e.g. imported drugs, drugs prepared by a pharmacy) or outside the terms of marketing authorization ("off-label"). In Switzerland, around half of all prescriptions for paediatric inpatients were either off-label or unlicensed [1].

Purpose To determine the proportion of unlicensed and off-label prescriptions at discharge, which has not been investigated previously, and the proportion of parents informed about such a prescription.

Materials and Methods Prospective study including all discharge prescriptions of inpatients over a two-month period at the Children's Hospital of Aarau. Exclusion criteria: hospitalisation for chemotherapy only, age over 18, re-entry during study period, no informed consent of parents. At discharge parents were asked to fill in a questionnaire about the information they got on discharge medicines as well as about their satisfaction with this information. This questionnaire was available in German, French, Croatian, Turkish, Albanian, Spanish and English.

Results During the study period 503 children were discharged, 231 children could be included. Discharge prescriptions were written for 140 children (61\%). A total of 227 drugs were prescribed, especially anti-inflammatory/analgesic, anti-asthmatic and anti-infective drugs. $38.5 \%$ of all prescriptions were off-label, regarding dosage in $51 \%$, age in $40 \%$ and indication in $9 \%$ of all cases. Only $0.5 \%$ of drugs were unlicensed. Discharge questionnaires were returned by 103 of 140 children. Most parents (>80\%) were informed about purpose, dosage and use of the drugs for their child, and satisfied with obtained information, but only $9 \%$ of parents getting an off-label/unlicensed prescription for their child were informed about the off-label/unlicensed use.

Conclusions There is a high percentage of drugs prescribed offlabel at hospital discharge. Most drugs are well known substances and regularly prescribed for children. This emphasises the need to update marketing information for older substances, or the need for a national database for drug use and dosage in children.

\section{Reference}

1. Paolo, E et al, 2006. Swiss. Med. WKLY. 136, 218-222.

No conflict of interest.

\section{BEA-006 USING LEADERSHIP TO TURN DEFEAT INTO SUCCESS}

doi:10.1136/ejhpharm-2013-000276.617

\section{A Forsstrom. Hospital Pharmacy at the University Hospital in Uppsala}

Background I was a manager at the Hospital Pharmacy at the University Hospital in Uppsala. The hospital has about 1100 beds. The hospital pharmacy employs a staff of about 75. Production consisted of both chemotherapy unit preparations and other sterile preparations. This Friday afternoon in November, I was summoned to the hospital's Chief Medical Officer. Once there, I receive complaints on the service from our production unit for Chemotherapy Preparations. Orders were often delivered too late - No notice of delays - Lack of communication between the hospital staff and the staff at the production unit.

Purpose To show how we improved the service from the Centralised Chemotherapy IV Preparation Unit.

Materials and Methods It was important to use my leadership to see something positive in what happened. It was not our skills that were complained about, but our service, which made it all easier. My point was, we are competent and skilled, now we have to improve our service. Together with the staff we decided:

- The complaint in terms of communication was true. Action: Hospital staff were invited.

- Tuesdays and Thursdays we had information for the staff, and we were always late. Action: We changed to providing more written information and just assembled the staff one Tuesday and Thursday every month.

- We did not answer the phone, or call and notify the delay. Action: We extended the staff in the morning by a technician who could answer the phone. Then we agreed to measure the delays. We completed a document in which we recorded when orders come in, if they were complete or if it needed a phone contact before they would be prepared. Then we documented when the preparations were ready. We decided to measure for 4 weeks in December. This measurement has since then been performed every year. We could pretty quickly see that we often received orders late and they were not complete.

Delivery of chemotherapy from Centralised Chemotherapy IV Preparation Unit at Uppsala University Hospital. Data for each year show the fraction of preparations delivered on time, fraction delivered after the requested time and the average time delay.

\section{Abstrtact BEA-006 Table 1}

\begin{tabular}{llllllll}
\hline Year & $\mathbf{2 0 0 5}$ & $\mathbf{2 0 0 6}$ & $\mathbf{2 0 0 7}$ & $\mathbf{2 0 0 8}$ & $\mathbf{2 0 0 9}$ & $\mathbf{2 0 1 0}$ & $\mathbf{2 0 1 1}$ \\
\hline Percentage delivered on time & $90 \%$ & $96 \%$ & $94 \%$ & $93 \%$ & $98 \%$ & $95 \%$ & $99 \%$ \\
Time delay & $19 \mathrm{~min}$ & $32 \mathrm{~min}$ & $16 \mathrm{~min}$ & $18 \mathrm{~min}$ & $32 \mathrm{~min}$ & $28 \mathrm{~min}$ & $36 \mathrm{~min}$ \\
\hline
\end{tabular}

These results were discussed later with the hospital staff and together we were able to improve the ordering schedule and we improved service with the deliveries. More preparations could be delivered on time.

Conclusions Inform your manager and staff. Take your time and plan how to handle the situation. Try to find out what you and your staff can learn. Try to understand the cause of the complaint and motivate staff for change. Try to measure in order to have a base for discussion and change. Good management is extra important for to support your staff when you and your staff are questioned.

No conflict of interest. 